\title{
Polarisation Characteristics of Pulsations Associated with Storm Sudden Commencements
}

\author{
Madhusudhan RAO, S. V. S. SARMA, and T. S. SASTRY \\ National Geophysical Research Institute, Hyderabad-7, India
}

(Received June 7, 1993; Revised December 15, 1993; Accepted January 26, 1994)

\begin{abstract}
Polarisation properties of magnetic pulsations associated with SCs recorded at an equatorial station, Choutuppal $\left(\mathrm{Gm}\right.$. Lat.: $\left.7.5^{\circ} \mathrm{N}\right)$, during the years $1970-88$ have been analysed. The results show that in contrast to a well defined local time dependent sense of rotation of polarisation vector reported for high and middle latitude pulsations (Wilson and Sugiura, 1961; Lanzerotti et al., 1981), the equatorial pulsations exhibit a predominantly clockwise sense of rotation particularly from around $22 \mathrm{hrs}$ to early afternoon hours ( 13 to $14 \mathrm{hrs} \mathrm{LT}$ ) while the events occurring later in the afternoon exhibit, in addition, a counter clockwise sense as well. The results for this equatorial station are consistent with the observations reported for very low latitude stations (Yumoto, 1986), thus indicating a latitudinal factor in characterising the local time dependence of sense of rotation of polarisation vector. The results are discussed in relation to the existing models invoking the azimuthally travelling ionospheric eddy currents for the immediate source of equatorial pulsations.
\end{abstract}

\section{Introduction}

One of the many interesting phenomena known to accompany sudden commencements of geomagnetic storms is the association of magnetic pulsations (Saito and Matsushita, 1967). Very few studies have been made on pulsations associated with storm sudden commencements and particularly so on their polarisation characteristics. The few studies made on polarisation characteristics of pulsations are mostly confined to the data from the middle and higher latitude stations. The present study is an attempt to deal with this aspect, namely the polarisation characteristics of, to start with, pulsations in the short period band (5-20 sec) associated with the SCs. The analysis is based on records of pulsations obtained at Choutuppal (Gm. Lat.: $7.5^{\circ} \mathrm{N}$ ), a station in the equatorial region of India situated about $60 \mathrm{~km}$ from Hyderabad. The records spanning a period of 19 years from 1970-88 form the data for this study. The data on SCs have been obtained from the LaCour magnetograms of the magnetic observatory at Hyderabad $\left(\mathrm{Gm}\right.$. Lat.: $\left.7.6^{\circ} \mathrm{N}\right)$ located on the campus of the National Geophysical Research Institute (NGRI).

\section{Data Analysis and Results}

The pulsations in the magnetic field are recorded in the three orthogonal components $H_{x}, H_{y}$ and $H_{z}$ at Choutuppal by means of an induction magnetometer set-up, where the signal outputs are amplified, integrated, filtered and finally recorded in analogue form on photographic paper in two period bands, viz. 0.5-20 sec and 20-1000 sec on separate recorders (for details please refer Sarma et al., 1969). The magnetic observatory at Hyderabad comprises a set of LaCour variometers for photographic recording of long period variations in the $H, D$ and $Z$ components of the geomagnetic field at chart speed of 15 $\mathrm{mm} / \mathrm{hr}$.

Being located in the equatorial latitudes the $H$ component of the geomagnetic field is the one that undergoes maximum variation among the three components and an SC of a storm is always registered at this station as a well defined rise in the $H$ (magnetic north) component. The magnetic observatory at Hyderabad has been in continuous operation since 1965 and Fig. 1 presents a few examples of SCs 
recorded here during the 19-year period (1970-88) under study.

One immediate feature that is apparent from visual inspection of the $\mathrm{LaCour}$ magnetograms from the Hyderabad magnetic observatory containing SCs and the corresponding magnetic pulsation records from the nearby Choutuppal observatory during the 19 year period, is that an overwhelming majority of the SCs are accompanied by pulsations.

About 80 well-defined SCs have been selected from the data and the magnetic pulsations corresponding to the above SCs were picked up from the Ultra Quick Run records (corresponding to the shorter period band) of the pulsation observatory at Choutuppal. In Fig. 2 are presented some examples of SCs and the corresponding pulsations (in the period range 5-20 sec) occurring in association with SCs. The arrows in this figure indicate the direction of progress of time on the LaCour and pulsations records. The SCassociated pulsations of this equatorial station would last only for a short time amounting to a few minutes.
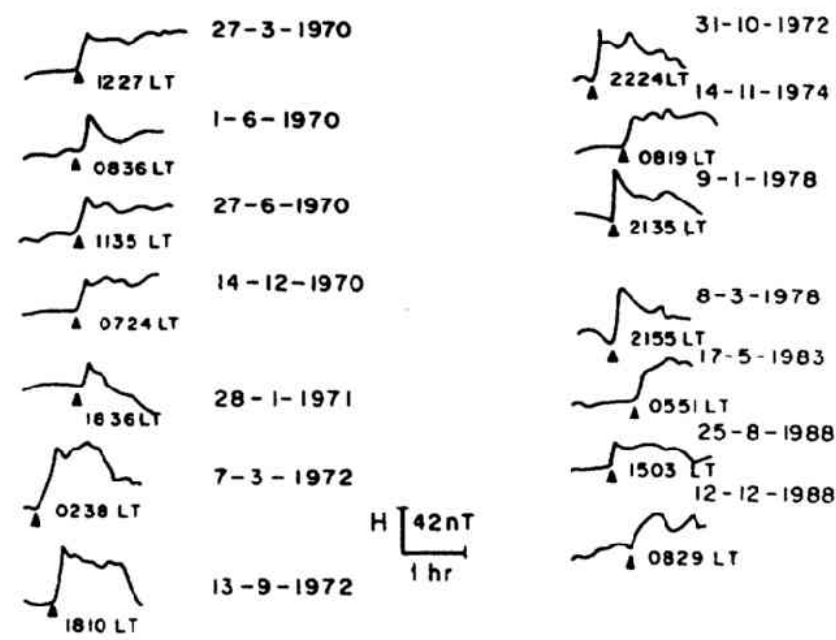

Fig. 1. Examples of storm sudden commencements recorded at Hyderabad $\left(\mathrm{Gm}\right.$. Lat.: $\left.7.6^{\circ} \mathrm{N}\right)$.

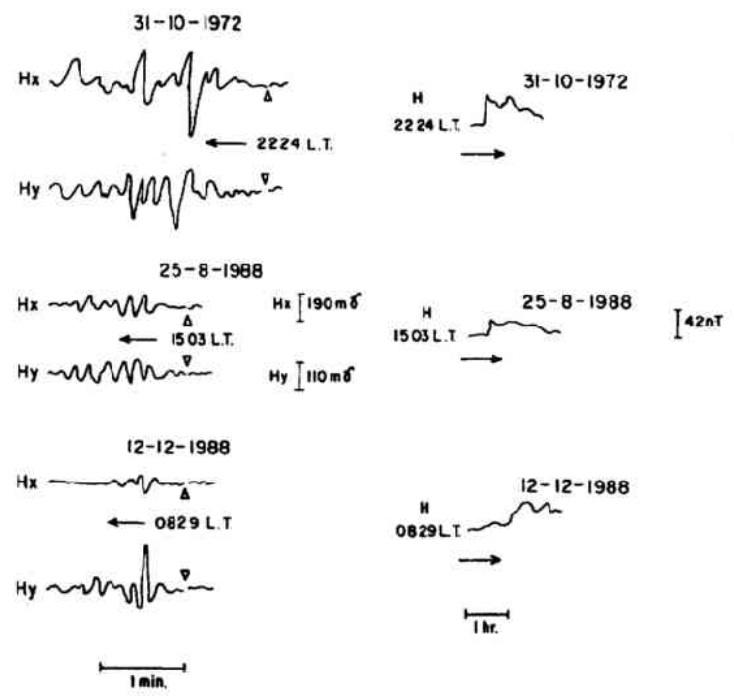

Fig. 2. Examples of $\mathrm{SC}$ associated short period pulsations along with the corresponding sudden commencements. 

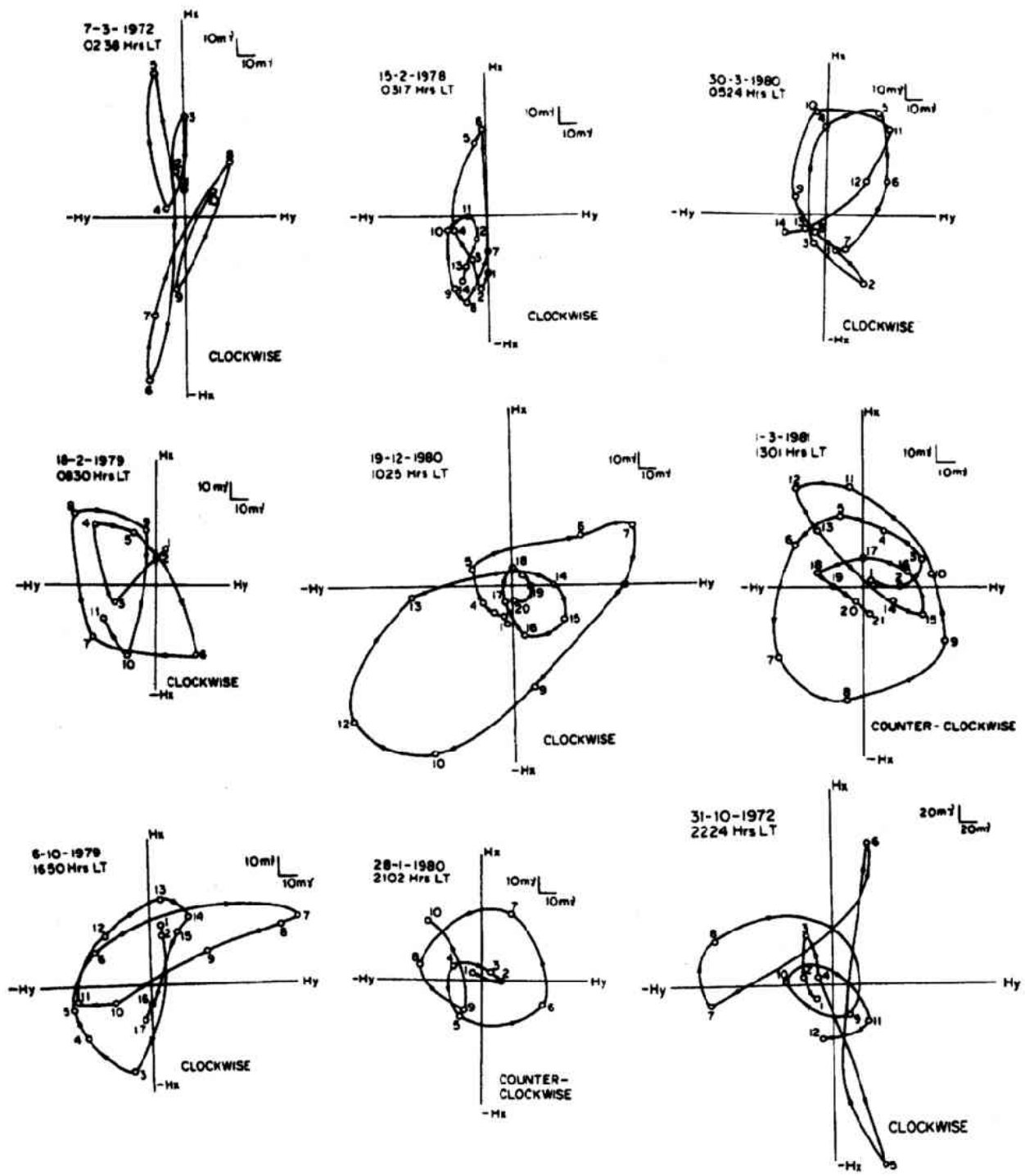

Fig. 3. Polarization diagrams of SC associated short period pulsations.

These short period pulsations in the $H_{x}$ and $H_{y}$ components have been hand-digitized at 2 sec interval and hodograms of horizontal vector are prepared for all the events. These are shown in Fig. 3 and are seen to be mostly elliptical with a few exhibiting circular polarisation.

The horizontal vector diagrams are examined for the sense of rotation of the polarisation vector. A histogram is prepared depicting the distribution of events of different senses of polarisation for each local time hourly interval (Fig. 4). The solid, shaded and dotted portions represent respectively the clockwise, counter-clockwise and mixed senses of polarisation with the ordinate showing the total number of events for each hourly interval and the abscissa the local time. The events show a clockwise sense of rotation for a major part of the daytime upto early afternoon hours, while the events during the rest of the day show, in addition, counter-clockwise (left-handed) or a mixed sense of rotation as well. Plots of horizontal vector made for the corresponding SC events (not shown) are found to be mostly of linear polarisation, with an occasional tendency to show a clockwise sense of rotation. 


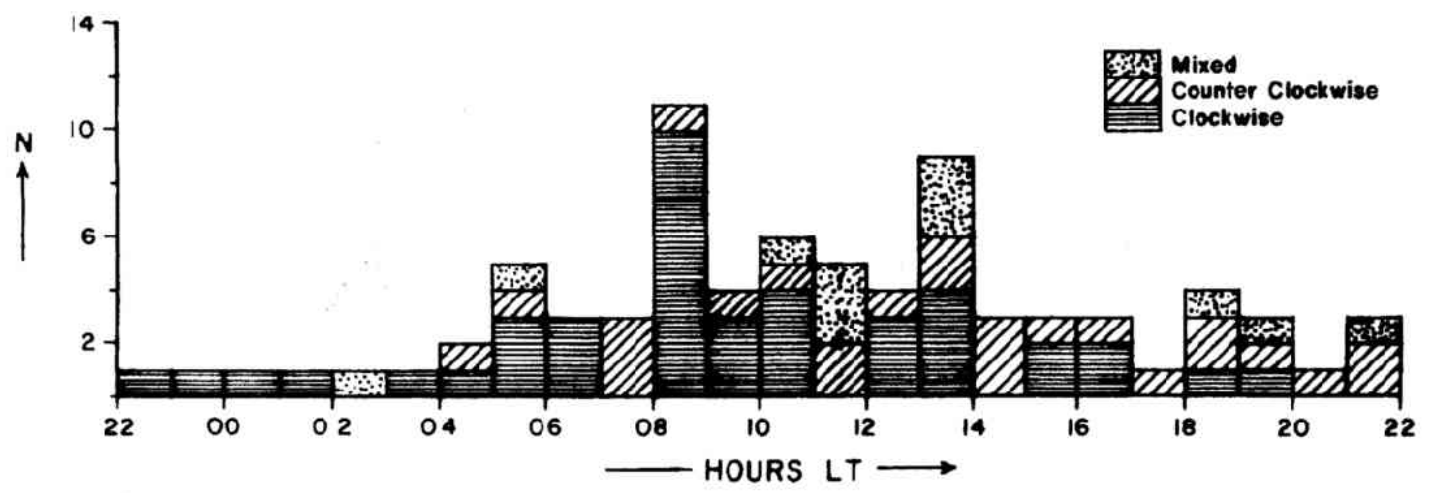

Fig. 4. Local time distribution of sense of rotation of polarization vector of $\mathrm{SC}$ associated short period pulsations at Choutuppal (Gm. Lat.: $\left.7.5^{\circ} \mathrm{N}\right)$.

\section{Discussion}

Wilson and Sugiura (1961) were among the first to report on the polarisation characteristics of SCs and the accompanying oscillations in the period range of a few minutes, corresponding to Pc4-5 pulsations, at middle and high latitude stations in the northern hemisphere. They have shown that the sense of rotation of polarisation vector for these pulsations shows a reversal across the 10-22 hr meridian - the sense of rotation which is dominantly counter-clockwise between 2200 and $1000 \mathrm{hrs} \mathrm{LT}$ becomes mainly clockwise between 1000 and $2200 \mathrm{hrs} \mathrm{LT}$.

Lanzerotti et al. (1981) and Yumoto et al. (1988) point out that the low latitude $(L=1.3-2.11)$ Pc3 pulsations show a polarisation reversal near local noon with a dominant forenoon counter-clockwise rotation changing to clockwise sense in the afternoon hours. However, coming down to further low latitudes $L=1.55$ and $L<1.11$ in the case of the regular Pc3 pulsations class not associated with storm sudden commencements, Yumoto (1986a) showed that the sense of rotation of polarisation vector which is predominantly right-handed (clockwise) before noon changes to a predominantly left-handed (counterclockwise) near local noon in the northern hemisphere. It is also pointed out that the change in the sense of rotation is not clearly defined in that the reversal occurs rather smoothly spreading over a few hours. This shows that $\mathrm{Pc} 3$ pulsations in very low latitude regions exibit a reversal in the polarisation sense across the noon meridian, with respect to that observed in middle latitudes. In addition, the switching over of the sense of polarisation is not well defined in the case of very low latitude stations.

Coming further down to the equatorial latitudes, as shown in the present study, the short period pulsations falling in the Pc3 period range and accompanying the storm sudden commencements also exhibit a local time variation of sense of rotation which appears to be broadly similar to that observed for regular Pc3 pulsations appearing at very low latitudes viz. dominantly clockwise upto around early afternoon hours and then gradually changing to a mixed or counter-clockwise along with a clockwise sense, during the afternoon hours.

Considering that the magnetic field lines anchoring below $22^{\circ}$ magnetic latitude do not go beyond the ionosphere Yumoto (1986) suggests the possibility of explaining the polarisation characteristics observed for regular Pc3 class of pulsations, by a system of azimuthally propagating ionospheric Pedersen currents induced by inductive electric field of the incoming compressional Pc3 waves. He also showed that the ionospheric eddy currents travelling in the westerly direction could account for the observed polarisation characteristics. Taking that this model of ionospheric eddy currents is valid for very low and equatorial latitude pulsations in general and in view of the broad similarity in the pattern of polarisation characteristics of short-period pulsations of the regular Pc2-3 class in the very low latitude region and those accompanying the SCs at Choutuppal as observed in the present study, it is suggested that the 
mechanism for their generation and propagation should also be similar. Accordingly, we may visualise that a compressional mode of $\mathrm{Pc} 3$ source wave would be generated at the magnetopause at the time of impact of shock waves corresponding to a storm sudden commencement and these waves after passing through the magnetosphere-ionosphere system produce corresponding eddy currents in the ionosphere. In view of the presence of clockwise rotation for a major part of the day, it may be inferred that the westward movement of these ionospheric eddy currents is more dominant for these events, in the equatorial region.

The authors are grateful to the Director, National Geophysical Research Institute, Hyderabad, for according permission to publish this paper.

The Editor thanks T. Araki and another referee for their assistance in evaluating this paper.

\section{REFERENCES}

Lanzerotti, L. J., M. V. Medford, C. G. Maclennan, T. Hasegawa, M. H. Acuna, and S. R. Dolce, Polarisation characteristics of hydromagnetic waves at low geomagnetic latitudes, J. Geophys. Res., 86, 5500-5506, 1981.

Saito, T. and S. Matsushita, Geomagnetic pulsations associated with SCs and SIs, Planet. Space Sci., 15, 573-587, 1967.

Sarma, Y. S., P. V. Sanker Narayan, K. R. Ramanujachary, S. V. S. Sarma, K. Yu. Zybin, and V. I. Makarov, Three component induction magnetometer for recording geomagnetic micropulsations at the geoelectric observatory, Choutuppal, Bull. NGRI, 7, 51-65, 1969.

Wilson, C. R. and M. Sugiura, Hydromagnetic interpretation of SCs of magnetic storms, J. Geophys. Res., 66, 4097-4112, 1961.

Yumoto, K., Generation mechanism of Pc 3 magnetic pulsations at very low latitudes, Planet. Space Sci., 34, 1329-1334, 1986.

Yumoto, K., T. Saito, Y. Tanaka, K. J. W. Lynn, F. W. Menk, and B. J. Fraser, Polarisation and amplitude characteristics of Pc3 pulsations in conjugate area around $L=1.3-2.1, J$. Geophys. Res., 93, 7386-7397, 1988. 